\title{
INTERNET A L'ÉCOLE : DU DISCOURS À LA MISE EN APPLICATION
}

\author{
Pascale Leroy ${ }^{1}$
}

\section{Introduction}

Alors que la télévision n'est toujours pas intégrée de façon véritable à l'école, et qu'une "éducation aux médias" des élèves n'est que rarement réalisée, de grands projets voient le jour en Belgique francophone, visant à équiper les établissements d'enseignement d'ordinateurs reliés à Internet. Ainsi, en Région wallonne, un programme fonctionnant depuis le $1^{\text {er }}$ septembre 1998 vise à installer dans un premier temps dans les écoles secondaires, puis dans les écoles primaires, des "Centres CyberMédia". Ces salles sont équipées de différents matériels informatiques, et une liaison avec Internet est prévue.

Dans ce domaine, cependant, la Ville de Bruxelles s'est montrée pionnière en lançant son propre projet, le projet "Brunette", dès la rentrée de septembre 1996, ce qui constituait une première en \footnotetext{
'Université Libre de Bruxelles, Centre de recherches sur le temps libre et les médias,
sous la direction de Roselyne BoulLLIN-DARTEVELLE.
} 
Belgique. Le plan d'équipement s'étalait sur cinq ans et est à présent en voie $\mathrm{d}$ 'achèvement.

A l'époque, et même encore aujourd'hui, l'idée de faire entrer une technologie nouvelle dans une structure aussi traditionnelle que celle de l'école était certainement audacieuse. Nous nous sommes demandée, dans les pages qui suivent, comment cette idée était née, quelles étaient les attentes des pouvoirs organisateurs, quels sont les usages réels dans les établissements d'enseignement, quelles sont les conséquences pédagogiques, quels bénéfices les étudiants peuvent en tirer...

Internet a valeur de mythe moderne : en interviewant les concepteurs du projet "Brunette" et en analysant leurs discours, nous voulions observer si ce projet était, dès le départ, suffisamment cohérent, et s'il s'insérait dans un ensemble de mesures plus structurelles et pédagogiques. Nous voulions également déterminer dans quelle mesure les décideurs s'étaient rendus compte qu'Internet était un (multi)média qui possédait, comme tous les médias, son langage et ses codes propres, à la lecture desquels professeurs et élèves devaient être formés.

Du mythe à la réalité : en recueillant les confidences des utilisateurs, nous désirions confronter les attentes des concepteurs avec la mise en application concrète dans les écoles.

La question essentielle était cependant de savoir si on ne risquait pas de simplement augmenter les compétences techniques des élèves sans réelle appropriation de l'outil. Nous avons donc cherché à déceler si une première appropriation avait ou n'avait pas eu lieu dans les écoles concernées, et quelles en étaient les raisons.

\section{Présentation du projet "Brunette" et de son site sur Internet}

En 1995, la Directrice générale du département de l'instruction publique de la Ville de Bruxelles convainc l'échevin de l'Instruction publique de la nécessité d'équiper tous les établissements scolaires dépendant du réseau de la Ville de Bruxelles d'ordinateurs reliés à Internet. L'échevin soutient le projet et le Conseil communal trouve l'enjeu suffisamment important pour voter, fait exceptionnel, un plan quinquennal. 
En septembre 1995, naît alors le projet "Brunette" (Brussels Network for Telematics in Education). Il s'agit, en cinq ans, de connecter à Internet toutes les écoles (du maternel au supérieur de niveau universitaire) du réseau de la Ville de Bruxelles, les bibliothèques, les centres psycho-médico-sociaux et de médecine scolaire, et différents services administratifs. De plus, ces divers sites doivent également être connectés entre eux.

Dans un premier temps, le service "Télématique et Communication" de l'Université Libre de Bruxelles s'occupe de la phase pilote du projet. Tout est à faire : déterminer les caractéristiques techniques du matériel, le type de connexion, visiter les établissements, estimer le coût... Peu avant la rentrée scolaire de septembre 1996, la phase 1 du projet peut cependant commencer : les dix premiers sites sont connectés et des formations à l'utilisation d'ordinateurs reliés à Internet sont mises en place, sur base volontaire, à destinations des professeurs et du personnel administratif. Depuis lors, la plupart des tâches assurées par le service "Télématique et Communication" ont été prises en charge par une équipe de la Ville de Bruxelles. Le projet est actuellement dans sa phase 3 (débutée en septembre 1998), et une quarantaine d'établissements ont été connectés. Il entrera dans sa dernière phase, la phase 5 , en septembre 2000.

Deux caractéristiques doivent dès l'abord être soulignées. Premièrement, toutes les écoles, bibliothèques... dépendant du réseau de la Ville de Bruxelles ont été connectées, de façon systématique, sans qu'elles doivent en faire la demande. Ensuite, au niveau technique, le projet "Brunette" apparaît comme très "ouvert" : les connexions sont permanentes, la liberté d'utilisation très grande, les contrôles fortement limités. Il se place donc dans une optique différente d'autres projets comme celui des "Centres CyberMédia", qui est fort directif (mise en armoires fermées du matériel, pas de traitement possible de documents trouvés sur Internet, détermination de logiciels admis...).

Un centre serveur d'informations, destiné aux écoles du réseau de la Ville de Bruxelles, a été mis en place ; "Brunette" dispose ainsi de son "site web", disponible à l'adresse suivante : "http://www.brunette.brucity.be". On y trouve notamment une liste des établissements d'enseignement et des bibliothèques ; un aperçu de différents projets pédagogiques en cours ou futurs ; un accès à des cours sur Internet ("Manuel d'utilisation de Netscape", "Introduction à l'HTLM"...), aux principaux moteurs de recherche, ou encore à des 
"coups de cœur" dans lesquels, matière par matière, différents liens renvoient à des sites intéressants, comme par exemple, le site du Louvre (avec possibilité de visite virtuelle) sous la rubrique "musées" ou les sites de divers quotidiens et chaînes de télévision sous la rubrique "journalisme".

La liste des écoles donne la possibilité de cliquer sur certaines d'entre elles et d'arriver ainsi sur "leur" site de présentation. Sur les 86 écoles ou implantations connectées (jusqu'en mars 1999), seules 33 utilisent cependant cette technique, avec un résultat très variable. Certains établissements se contentent de renvoyer simplement à une demi-page de présentation contenant... leur adresse, leur numéro de téléphone et le nom des directeurs. La plupart mettent sur leur site une série de renseignements que l'on peut trouver par ailleurs dans des brochures imprimées : photo du bâtiment, heures d'ouverture, spécificités des différentes sections, projet pédagogique, etc.; la recherche graphique est limitée. Seul un petit nombre d'écoles utilisent réellement toutes les richesses permises par ce genre de média ; même dans ce cas, cependant, il n'y a pas mention explicite d'Internet et de ce que cette nouvelle technique peut apporter.

Introduire Internet à l'école, serait-ce finalement un rêve de technocrate ? Ce projet est-il réalisable ? Et qu'en pensent les acteurs de terrain confrontés à son application? Pour répondre à ces questions, nous avons mené une enquête que nous détaillons cidessous.

\section{Description de l'enquête réalisée}

Nous avons rencontré et interviewé les principaux responsables du projet "Brunette", à savoir Madame Verrept, Directrice générale du département de l'instruction publique de la Ville de Bruxelles, Monsieur Nys, responsable informatique du projet, Madame Paroni, chercheur au service "Télématique et Communication" de l'Université Libre de Bruxelles et responsable de la phase pilote du projet, et Madame Messori, responsable "pédagogique" de la phase pilote.

Nous nous sommes rendue dans deux établissements d'enseignement secondaire (enseignement général), le Lycée Émile Jacqmain et l'Athénée Adolphe Max. Au Lycée Émile Jacqmain, nous avons interviewé la Préfète, Madame Marneffe ainsi que les responsables du site Internet de l'école, Messieurs Vancabeke (professeur de mathé- 
matiques) et Van Osselt (secrétaire). Nous avons pu suivre une partie du déroulement d'un des projets pédagogiques soutenus par "Brunette", le projet "Parleunet", interviewé son responsable pour l'école, Monsieur Van Strijtem (professeur d'anglais), et soumis un questionnaire aux élèves qui participent au projet. A l'Athénée Adolphe Max, nous avons interviewé le Préfet, Monsieur Poels, Monsieur Meylemans (professeur de géographie) qui anime une activité parascolaire, le "Club Internet" (ici aussi, les élèves de ce club ont rempli un questionnaire), et Monsieur Boumal (professeur de français) qui anime une autre activité parascolaire, le "Club Multimédia".

Cette enquête ne prétend à aucune exhaustivité. Elle reflète bien, cependant, à notre avis, la situation dans les autres établissements. Une étude systématique reste néanmoins à réaliser.

\section{Le long chemin vers l'appropriation}

\section{a. Internet à l'école est une innovation}

A la base du projet "Brunette", se trouve clairement "une volonté politique de câbler toutes les écoles" (Alain Nys). Nous l'avons déjà signalé : tous les établissements sont connectés, sur une base géographique, et de façon systématique. Même si des séances d'information et de concertation ont été organisées avec les inspecteurs et chefs d'établissements durant la phase préparatoire, l'idée vient de l'administration. Ceci constitue un cas très intéressant d'une mise à disposition d'une innovation technologique, sans que les utilisateurs pressentis en aient exprimé le désir.

La date choisie pour le début de la phase pilote n'est pas fortuite. A la fin de l'année 1995, l'enseignement en Belgique francophone est en pleine "période de tourmente à la fois idéologique, pédagogique et budgétaire". Le gouvernement cherche les moyens de faire des économies, professeurs et politiques aspirent à un autre type de pédagogie, les enseignants et les élèves, inquiets pour leur avenir, ont derrière eux plusieurs grandes grèves mobilisatrices. Dans ce contexte, l'introduction d'Internet et de ses nombreuses applications éducatives au sein des écoles apparaît comme une solution possible à

\footnotetext{
${ }^{1}$ Cliquez net !, Bruxelles, Média Animation, 1997, p. 11.
} 
tous ces problèmes : "remotiver les enseignants" (Monique Verrept), "redonner un essor à l'enseignement" (Monique Verrept), "former les nouveaux citoyens" (Nadia Paroni) sont quelques-uns des buts recherchés. Cette idée d'une nouvelle technologie salvatrice n'est pas propre à la Belgique, comme le montre un rapport de l'OCDE sur l'introduction des nouvelles technologies de l'information dans le monde de l'éducation: "son avènement [d'une nouvelle technologie] coïncide avec une période de crise pour la plupart des systèmes éducatifs"1. Avec le danger de "replacer les technologies dans le contexte de la crise, c'est-à-dire de n'en voir que les aspects susceptibles de contribuer à résoudre la crise, au lieu de les situer dans le contexte national de l'éducation"'.

Si parmi les différentes technologies de l'information, Internet s'est imposée dans le projet "Brunette", c'est à nos yeux principalement grâce à trois de ses caractéristiques.

Premièrement, il s'agit d'une nouveauté technologique dont les usages ne sont pas encore fixés. Cette grande plasticité permet à chacun d'y investir ses fantasmes et d'y chercher ce qu'il désire, puisque tout est théoriquement possible.

Ensuite, et cette caractéristique découle de la première, même si les applications futures d'Internet échappent encore en grande partie à toute prospective sérieuse, même si personne ne peut assurer avec certitude où cela va mener, chacun veut y être avant les autres. Dans le cas du projet "Brunette", les responsables de la phase pilote ont ainsi clairement ressenti le désir du pouvoir organisateur d'être "les premiers", de posséder les "premières écoles en Belgique" raccordées à Internet. Au-delà d'une éventuelle question de prestige, ceci correspond parfaitement à ce que Dominique Wolton qualifie de "logique de l'urgence". Cette nouvelle technologie doit être intégrée rapidement à l'école, sous peine que les jeunes se retrouvent "à la traîne" (Nadia Paroni), comme le confirment la plupart des "futurologues" actuels : "décideurs et enseignants [doivent] attraper le train en marche"3, même si personne ne sait pour quelle destination ; l'important est "de

${ }^{1}$ Les nouvelles technologies de l'information - Un défi pour l'éducation, Paris, OCDE (Organisation de coopération et de développement économiques), 1986, p. 19.

${ }^{2}$ Ibid.

${ }^{3} \mathrm{R}$. COHEN, La communication télématique internationale. Une mutation dans l'éducation? Des expériences à travers l'Europe, Paris, Retz, 1995, p. 9. 
ne pas être laissés sur le quai de gare"1. Comme le fait très justement remarquer Dominique Wolton, "le décalage est toujours considérable entre le caractère inévitablement approximatif des prévisions, et la manière définitive avec laquelle on conclut au caractère impératif de telle ou telle politique à mener"'2.

Troisièmement, Internet procède de ce que l'on pourrait appeler un sentiment d'évidence. Il est tellement "évident" qu'il faut introduire Internet dans le monde éducatif que cela semble dispenser de se fixer des buts et des objectifs précis, comme si l'introduction de cette nouvelle technologie était un but en soi. La notion même d'"Internet" apparaît comme mythique, presque "divinisée", échappant de ce fait aux lois terrestres. Or, il ne s'agit rien de plus qu'une innovation qui va devoir, au fil du temps, comme toutes les autres innovations, être adoptée au sein d'un certain milieu (ici, l'école), s'y adapter et s'y forger des usages.

\section{b. Le discours techniciste}

Ces différents phénomènes ont été abondamment étudiés par la sociologie de l'innovation. Comme son nom l'indique, ce courant de la sociologie s'intéresse à tous les processus qui sont à l'œuvre lors de la diffusion et l'adoption d'une innovation parmi la population. Lorsque les scientifiques ont commencé à étudier le problème, ils avaient de l'innovation technologique une vision très linéaire. Voici par exemple comment on a défini le processus lors de l'Exposition universelle qui s'est tenue à Chicago en 1933: "la science découvre, l'industrie applique, l'homme suit". Cette approche techniciste, qui a aussi été appelée "déterminisme technique" postule que c'est l'innovation technologique en elle-même qui détermine et structure les usages qui en sont fait. Dès les années 40 cependant, des sociologues américains qui veulent comprendre dans quelles conditions de nouvelles techniques agricoles sont adoptées par les fermiers se rendent compte que la question de l'innovation technologique est beaucoup plus complexe. C'est le début d'une longue remise en cause de l'approche techniciste basée sur l'observation d'une "absence

\footnotetext{
1 Ibid.

2 D. Wolton, Penser la communication, Paris, Flammarion, 1997, p. 265.
} 
d'homothétie entre les changements technologiques et les changements sociaux" 1 .

Ceci n'empêche cependant pas ce discours techniciste de demeurer très présent chez la plupart des techniciens et des politiques ; le constat est particulièrement frappant dans le cas des nouvelles technologies de l'information et de la communication.

C'est surtout dans la littérature non scientifique -essais, textes de vulgarisation, articles journalistiques et documents promotionnels des producteurs de technologies-que le schème déterministe est le plus présent. Lorsqu'on nous décrit la façon dont les nouvelles technologies de communication (NTC) vont bouleverser notre vie, changer notre représentation du monde ou notre rapport aux autres, (par exemple favoriser un repli de l'individu sur la sphère privée), c'est bien une conception déterministe de la technologie qui est en ouvre. Alors même que ces NTC sont encore en gestation, on postule que celles-ci vont entraîner un type d'usages précis et connus à l'avance, comme si les machines à communiquer impliquaient, du fait de leur configuration, un et un seul modus operandi, comme si leurs usagers n'avaient aucune capacité d'action sur celles-ci².

Pierre Chambat évoque de même le "mélange de discours techniciste et de prophétie qui caractérise trop souvent les revues professionnelles quand elles abordent les usages" 3 . Dominique Wolton parlant quant à lui de "fuite en avant technologique" : "pour le moment, l'offre est largement en avance sur la demande (...). Mais au lieu d'admettre cette incertitude, l'idéologie technique fait au contraire comme si les besoins allaient naturellement se développer"4.

Dans le cas de "Brunette", les propos tenus par plusieurs responsables du projet procèdent parfois de cette approche déterministe : "si on donne aux enseignants quelque chose de motivant [Internet], cela va les redynamiser" (Monique Verrept), "le but était que chaque école développe son projet" (Alain Nys), ou encore "au départ, les formations étaient surtout techniques" (Nadia Paroni). L'idée d'une

1 P.-A. Mercier, Fr. Plassard, V. Scardigli, Société digitale. Les nouvelles technologies au futur quotidien, Paris, Éd. du Seuil, 1984, p. 179.

2 Th. VEDEL, "Sociologie des innovations technologiques et usagers: une introduction à une socio-politique des usages", in Médias et nouvelles technologies. Pour une socio-politique des usages, Rennes, Éd. Apogée, 1994, pp. 23-24.

${ }^{3}$ P. CHAMBAT, "NTIC et représentation des usagers", in Médias et nouvelles technologies, op. cit., p. 45.

${ }^{4}$ D. WOLTON, op. cit., p. 241. 
technique agissante est très présente : si on fournit une nouvelle technologie, quelques orientations très générales, et la possibilité d'une formation essentiellement technique, les écoles sont censées insérer cette nouvelle pratique dans leur structure, et les enseignants modifier leurs pratiques pédagogiques.

\section{c. De l'observation à l'appropriation}

En réalité, la sociologie de l'innovation a montré que les utilisateurs des systèmes techniques disposent d'une large autonomie dans la détermination des usages, même si ceux-ci sont limités par une série de facteurs sur lesquels nous reviendrons plus loin.

Si on se place du côté du consommateur des nouvelles technologies, on observe que le processus qui va mener aux usages définitifs et à une éventuelle appropriation de l'innovation se déroule en trois étapes.

\section{- La première étape est celle de l'observation.}

Les consommateurs observent l'innovation technologique et, dans un premier temps, vont simplement reproduire grâce à celle-ci des pratiques antérieures ${ }^{1}$.

Ceci explique le fait que l'introduction d'Internet à l'école ne signifie pas automatiquement que cette nouvelle technologie va être utilisée d'une manière innovante, génératrice de nouvelles pratiques pédagogiques. Les anciennes pratiques demeurent, voire même s'affirment avec plus de prégnance encore grâce au nouvel "outil"2. Le même phénomène a d'ailleurs été rencontré lors des diverses tentatives d'introduction de l'audiovisuel à l'école. L'analyse qu'en fait Geneviève Jacquinot est particulièrement éclairante : "l'audiovisuel a trop longtemps été (...) conçu comme autonome, comme un outil technique (...) propre à faire «plus vite», «pour plus de gens», «avec plus d'efficacité», ce l'on avait toujours fait avant. Adjuvant neutre, prothèse électronique... les auxiliaires audiovisuels ont le plus souvent renforcé les pratiques pédagogiques existantes"3. Il s'agit de

1 Voir à ce sujet P.-A. MERCIER et al., op. cit, pp. 176-177.

2 G. DeLACOTE, Savoir apprendre. Les nouvelles méthodes, Paris, Odile Jacob, 1996, p. 35.

3 G. JACQUNOT, L'école devant les écrans, Paris, Les Éd. ESF, 1985, pp. 39-40. 
ne jamais confondre "innovation technique" et "innovation pédagogique"1.

Il est intéressant de noter que dans les deux établissements bruxellois que nous avons visités, les ordinateurs en eux-mêmes sont plus employés que les connexions Internet. Ainsi, au Lycée Émile Jacqmain, l'arrivée de nouveaux ordinateurs a permis la création d'un cours de technologie (utilisation de logiciels, CD Roms...) en première et deuxième année. A l'Athénée Adolphe Max, les ordinateurs permettent aux élèves de manipuler plus facilement des mesures physiques grâce au tableur "Excel" et de réaliser la mise en page du petit journal de l'école; le préfet reconnât d'ailleurs volontiers qu'“on utilise plus les ordinateurs fournis qu'Internet", ajoutant qu' 'il faut trouver le bon moyen d'utiliser Internet (...) mais d'abord il faut bien l'observer".

- La deuxième étape vers l'appropriation découle de la première : c'est celle de la comparaison.

Dans le cadre des anciennes pratiques, les utilisateurs vont comparer la nouvelle technologie à l'ancienne et en soupeser les avantages éventuels. Ce processus ne s'effectue pas in abstracto; pour juger une innovation, un individu détermine d'abord si celle-ci est en concordance avec ses besoins ainsi qu'avec ses attitudes et croyances $^{2}$. Les auteurs d'un rapport sur un programme belge de recherche sur les conditions et les implications de l'innovation technologique ${ }^{3}$ arrivent à une conclusion semblable: "il n'y a demande et usage effectif d'un support technologique que lorsque celui-ci répond à un besoin et présente en outre un avantage comparatif net par rapport à d'autres moyens de répondre à ce besoin"4.

${ }^{1}$ Voir à ce sujet A. GRYSPEERDT, Usages scolaires des médias. Les médias à l'école: vidéo, bandes dessinées, presse écrite, jeux, informatique..., Louvain-la-Neuve, Cabay, 1983, p. 258.

${ }^{2}$ Voir à ce sujet E. M. Rogers, F. F. SHOEMAKER, Communication of Innovation. A cross-cultural Approach, New York, The Free Press, 1971, p. 105.

${ }^{3}$ Le programme FAST (en référence au programme européen de même nom) qui s'est déroulé de 1984 à 1987.

${ }^{4}$ Actions nationales de recherche en soutien à FAST. Rapport final, Bruxelles, Services du premier ministre, Services de programmation de la politique scientifique, 1988, p. 257. 
N'oublions pas que l'innovation arrive dans un certain type d'école, avec certains professeurs et certains élèves, qui ont tous leur "histoire", leur éducation, leur façon de percevoir le monde.

Lors de l'analyse du projet "Brunette", nous avons été frappée par les attitudes "attentistes" des directions d'école et sceptiques des enseignants. Ainsi, les préfets se retrouvent un peu dans la peau de non-fumeurs à qui on aurait offert des cigares cubains : ils apprécient le "cadeau" à sa juste valeur... mais ne savent pas très bien quoi en faire. Chacun (préfets, professeurs, responsables du projet) insiste sur le fait qu'Internet est un "outil"; ce mot revient de façon lancinante dans toutes les interviews. On demande donc à cet "outil" de remplir d'anciennes fonctions et on compare ses aptitudes à celles des anciennes techniques. On le compare au tableau noir ou au cahier (traitement de texte), à l'échange de lettres par la poste (e-mail), à la recherche en bibliothèque (recherche on-line)... Les problèmes techniques et de manipulation aidant, les avantages de la nouvelle technologie ne sont pas considérés comme révolutionnaires. Nous verrons plus loin qu'une réelle appropriation d'Internet ne peut avoir lieu que si l'on cesse de ne le considérer que comme un "outil".

- A ce stade, si la comparaison entre l'ancienne et la nouvelle technologie n'est pas favorable à cette dernière, l'innovation peut ne plus être utilisée et tomber dans l'oubli. Autrement, les consommateurs vont, lors d'une troisième étape, procéder à l'établissement progressif de nouveaux usages de l'innovation, détachés des anciennes pratiques. Néanmoins, il s'agit de ne pas être dupe et de ne pas passer d'une approche techniciste (où les concepteurs déterminent les usages futurs d'une innovation) à une approche "consummériste" (où les consommateurs seraient entièrement libres de déterminer leurs propres usages). Une nouvelle approche, développée au début des années 90 , emprunte une voie médiane en tentant de replacer les usages au sein de leur contexte, c'est-à-dire à l'intérieur de la société tout entière : il s'agit de la "socio-politique des usages". "L'usage apparaît en effet au croisement de trois logiques principales : une logique technique qui définit le champ des possibles, une logique économique qui détermine le champ des utilisations rentables et une logique sociale qui détermine la position particulière du consommateur avec ses besoins et ses désirs"1.

\footnotetext{
1 A. Vitalis (dir.), Médias et nouvelles technologies, op. cit., p. 9.
} 
L'école est donc bien plus qu'un simple réceptacle pour des usages imaginés ailleurs. Même si elle est fortement insérée dans une société, sa capacité d'action sur le présent -et surtout sur le futurdemeure importante :

On pourrait dire que la société est dans son école: nous faisons partie intégrante d'un système qui nous fait autant que nous le faisons. L'attitude que l'école prendra au regard des nouvelles technologies ne sera pas sans effet sur la place que celles-ci auront demain, dans notre quotidien (...). L'école peut être à l'origine de la création de nouveaux projets sociaux 1 .

Elle pourrait donc, plutôt que de les subir passivement, influencer les usages futurs des nouvelles technologies. Tant il est vrai que "la technique seule ne donnera pas du sens à la société de la communication de demain"2. Et quel meilleur endroit que l'école pour réfléchir à cette question essentielle du sens ?

C'est seulement à ce moment que l'on pourra réellement parler d'une appropriation d'Internet par les professeurs et les élèves.

La plupart des établissements bruxellois ont franchi la première et la deuxième étape menant à ce but, mais nous n'avons pas encore décelé de véritable appropriation du multimédia.

\section{Les raisons du manque d'appropriation}

Les raisons en sont qu'on ne peut imaginer de nouveaux usages à une technologie ni opérer une réflexion sur le "sens" à donner à celleci que lorsque deux conditions sont remplies au sein de l'école : l'insertion de l'innovation dans un projet pédagogique global et l'existence d'une éducation au (multi)média. Ce n'est qu'à ce moment-là qu'on pourra observer une appropriation d'Internet. En effet, s'il n'y a pas de projet pédagogique global, on risque d'assister à une simple reproduction des anciennes pratiques. Et s'il n'y a pas d'éducation aux médias, la réflexion sur le sens aura toutes les

1 G. BRAUN, M. QUERE, "Nouvelles pratiques d'enseignement", in Enseignement, formation et nouvelles technologies, Dossiers de l'Audiovisuel, $\mathrm{n}^{\circ} 75$, sept./oct. 1997, p. 9.

2 Conférence de Dominique Wolton dans le cadre d" Initiatives ", "Forum de l'entreprise en Belgique francophone ", Halles des Foires de Liège, 16 oct. 1998. 
chances d'être évacuée et seuls les usages imaginés par les concepteurs ou voulus par le monde économique s'implanteront.

\section{a. Un projet pédagogique global}

Ce à quoi on a assisté jusqu'à présent est à une introduction d'Internet pour Internet, cette nouvelle technologie étant considérée comme une fin en soi. Or, "échanger, communiquer, voilà de nobles intentions, mais il convient d'aller plus loin, en particulier dans la définition des objectifs éducatifs que l'on assigne à ces activités. (...) Si l'on souhaite éviter les désillusions et les échecs, il convient de faire avancer d'un même pas les équipements et les usages (...)"1.

Comme nous le confiait le responsable du "Club Multimédia" du Lycée Émile Jacqmain à propos de l'accès au "World Wide Web": "la mission de l'école n'est pas, sauf cas d'espèce, de faire de la consultation des réseaux électroniques une finalité en soi" (Michel Boumal) et à propos de la pratique du courrier électronique par les élèves : "ça ne sert à rien de faire de la communication si on n'a rien à dire".

Un livre écrit en 1988 et qui traite notamment de l'enseignement assisté par ordinateur apparaît comme visionnaire lorsqu'il prétend que "les butoirs de l'informatique de formation ne seront plus, demain, les problèmes techniques ou les résistances sociologiques ou psychologiques à l'utilisation de l'informatique mais les insuffisances de la réflexion pédagogique"2.

Pour qu'un projet pédagogique global puisse être présent dans l'école, afin que les nouvelles technologies puissent être utilisées de façon innovante, et pas comme des "gadgets", les trois éléments suivants sont indispensables :

\section{- Un projet}

Nous avons déjà évoqué la grande plasticité d'Internet, ses usages n'étant pas encore définitivement fixés. Chaque intervenant aura donc ses raisons propres de justifier l'introduction d'Internet. Une justification qui revient régulièrement est celle de la préparation des élèves à un monde futur ("former les nouveaux citoyens", Nadia

\footnotetext{
1 "Échanger, communiquer... oui, mais quoi et avec qui ?", in Internet dans le monde éducatif, Les dossiers de l'ingénierie éducative, $\mathrm{n}^{\circ} 24$, déc. 1996, pp. 25 et 27.

2 Y. Bourron, J.-P. Chapuis, Chr. Canonville, P. Colin, Audiovisuel, mode d'emploi. Langage et pratique, Paris, Les Éd. d'Organisation, 1988, p. 181.
} 
Paroni, "auto-formation des citoyens", Nathalie Messori...). Une brochure de présentation du projet "Brunette" réalisée par le Service "Télématique et Communication" en évoque une multitude d'autres :

La création d'information sur Internet peut être une activité ludique, éducative et particulièrement motivante pour les jeunes : en effet, ils peuvent s'y exprimer, faire connaître leurs aspirations et leurs besoins, s'ouvrir à de nouvelles langues, à d'autres cultures. (...) [Ils] sont exposés à une publicité planétaire, ce qui les incite à soigner leurs productions ${ }^{1}$.

Il s'agit ici d'un bel exemple d'un ensemble d'affirmations péremptoires à caractère visionnaire ; l'accès à Internet est considéré comme une baguette magique qui va tout à la fois amuser les élèves, les instruire, les motiver, améliorer leur connaissance des langues et leur orthographe, les rendre plus tolérants et remplir une saine fonction de psychothérapie.

Lorsqu'on interroge les responsables du projet sur les buts poursuivis, les réponses sont assez vagues. On se rend compte que les questions de base n'ont pas été posées : quelles sont les caractéristiques de ce "monde futur" auquel les étudiants doivent se préparer, que recherche-t-on exactement via l'introduction d'Internet, et surtout, dans quel projet cela va-t-il s'insérer?

En réalité, un véritable projet porteur pourrait être de considérer Internet à la lumière des recommandations politiques récentes sur l'orientation à donner à l'enseignement. On y parle en effet d'un enseignement qui doit "promouvoir le développement de la personne de chacun des élèves", "en amenant les jeunes à construire leur savoir, les conduire à prendre une place active dans la vie économique", "amener les jeunes à être des citoyens responsables dans une société libre", ou encore "amener tous les élèves à s'approprier des savoirs et acquérir des compétences qui les rendent aptes à apprendre toute leur vie et à prendre une place active dans la vie économique, sociale et culturelle"3. On voit que les potentialités offertes par Internet pourraient contribuer à la réalisation des objectifs actuels assignés à

1 Université Libre de Bruxelles - Service Télématique et Communication, Le projet Brunette (Brussels Network for Telematics in Education), févr. 1997, p. 2.

2 Extraits de : Conseil de l'Éducation et de la Formation, Les objectifs du système d'enseignement et de formation, 5 févr. 1992.

3 Extrait de : Communauté française, Les missions prioritaires de l'enseignement fondamental et de l'enseignement secondaire, décret du 24 juillet 1997. 
l'éducation. Tout ceci risque cependant de ne rester qu'au stade du discours tant que ce projet porteur ne peut s'appuyer sur une pédagogie elle aussi renouvelée.

\section{- Une pédagogie nouvelle}

En effet, "la nouvelle technologie de l'information offre au système éducatif une alternative, dans la mesure où elle se présente comme le véhicule qui intégrera quelques-uns des principes depuis longtemps familiers aux esprits novateurs, mais qui n'ont que récemment acquis du crédit grâce aux travaux de recherche sur la cognition. (...) L'obstacle principal est la résistance de l'institution scolaire à tout changement d'organisation tendant à substituer à l'enseignement (au discours magistral) comme origine du savoir, l'apprentissage fondé sur la manipulation et l'expérimentation de ses objets"'. De plus en plus de pédagogues sont d'accord pour promouvoir un enseignement qui devrait "centrer les méthodes de travail sur l'apprenant mis en situation d'investigation et d'analyse plus que sur la transmission de contenus fixés"'2. Sans cette nouvelle pédagogie, les bénéfices engrangés par l'utilisation d'Internet seront maigres, voire inexistants, ce qui a pu amener le "Centre de ressources en éducation aux médias et au multimédia" (responsable pour l'enseignement catholique) à déclarer dans un livre publié tout récemment que "dans le cadre d'une pédagogie traditionnelle, l'outil [Internet] est inutile"3. Ce qui explique tout à fait l'attitude sceptique des enseignants censés utiliser cette nouvelle technologie.

Les concepteurs du projet "Brunette" nous ont apparu tenir à ce propos un discours ambigu. D'une part, ils promeuvent clairement l'avènement d'une nouvelle manière de travailler, permise grâce aux potentialités d'Internet : "l'éducation et l'enseignement sont rénovés grâce aux technologies modernes", ou "régulièrement, il faut essayer

1 Les nouvelles technologies de l'information, op. cit., pp. 74 et 89.

2 G. JACQUINOT, "La télévision : de l'outil pédagogique à la pédagogie de l'outil ", in Actes du colloque international - La télévision : un outil pédagogique pour les jeunes citoyens européens?, Bruxelles, Parlement européen, 26 et 27 nov. 1993, p. 62.

${ }^{3}$ Du tableau à la toile. Découverte du multimédia pédagogique, Bruxelles, Média Animation (Centre de ressources en éducation aux médias et au multimédia), août 1998, p. 47.

4 Extrait de : "Le projet éducatif de la Ville de Bruxelles", année scolaire 19981999. 
de montrer que les matières ne sont pas cloisonnées" (Monique Verrept). D'autre part, les politiques espèrent que l'innovation pourra s'imposer sans heurt, sans toucher au fonctionnement classique de l'enseignement, ce qui fait dire à Dominique Nora que "la difficulté n'est pas tant l'argent disponible pour équiper les écoles que la capacité de nos sociétés à réformer leur système d'enseignement"1. Cette tendance semble se confirmer dans tous les pays de l'OCDE confrontés au problème : “(...) tous les politiques partent de l'hypothèse tacite que l'enseignement ne changera pas de visage, et que l'introduction de la nouvelles technologie de l'information peut être planifiée sans avoir à modifier la structure du système"2. La directrice générale du département de l'instruction publique, Monique Verrept, relève d'ailleurs immédiatement la contradiction en affirmant que "le ministre nous dit qu'il faut décloisonner les matières, mais d'un autre côté, il y a les programmes à suivre". Or, sur le terrain, la structure géographique et hiérarchique de l'école, son fonctionnement horaire, ses modes d'évaluation, restent inchangés, rendant la mise en œuvre d'un audacieux projet comme "Brunette" difficilement réalisable.

L'application d'une pédagogie rénovée dépasse d'ailleurs largement le simple cas d'Internet. Ainsi, diverses expériences ont souligné les nombreux aspects positifs du travail scolaire avec cette nouvelle technologie : on parle volontiers de "développement de la motivation", "développement de l'entraide et de la communication", "développement de l'autonomie et de l'intiative", ou "changement d'attitude à l'égard de la langue écrite" ${ }^{\text {. }}$. On peut cependant se poser la même question quelque peu iconoclaste que le "Centre de ressources en éducation aux médias et au multimédia" en se demandant si "ce n'est pas la machine qui est la cause première de cette situation pédagogique, mais bien le choix pédagogique de l'enseignant" ${ }^{\prime 4}$. Mais ceci est encore un autre problème...

\section{- Un nouveau rôle pour les enseignants et les élèves}

Cette pédagogie nouvelle demande évidemment que les enseignants y soient formés et qu'ils acceptent de remplir un nouveau rôle au sein de leur classe. L'exposé magistral ne serait plus la norme

' D. NORA, Les conquérants du cybermonde, Paris, Calmann-Lévy, 1995, p. 368.

2 Les nouvelles technologies de l'information, op. cit., p. 29.

${ }^{3}$ R. COHEN, op. cit., pp. 50-51.

4 Du tableau à la toile, op. cit., p. 48. 
comme c'est le cas aujourd'hui ; il serait remplacé par des activités au cours desquelles les élèves participent de façon active à leur éducation, l'enseignant devenant dès lors plutôt un "guide", une "personneressources".

Lorsqu' on passe à une application sur le terrain, on retrouve cependant la même problématique que celle dont nous avons parlé à propos de la nouvelle pédagogie, à savoir l'ambiguitté de la position du pouvoir organisateur : l'enseignant devrait tantôt remplir un rôle traditionnel (programmes à suivre, homologation...), tantôt se muer en "animateur" donnant accès à ses élèves à un autre mode d'apprentissage. Ainsi, l'enseignant devrait utiliser Internet dans ses cours, mais la structure dans laquelle il doit réaliser ce projet (heures de cours, géographie des classes...) ne change pas, elle. Ou encore, l'enseignant devrait continuer à se former durant toute sa carrière, échanger des informations avec d'autres enseignants du réseau "Brunette", voire du monde entier: "le projet «Brunette» vise le décloisonnement des enseignants" (Monique Verrept), "tous les professeurs de biologie, par exemple, pourraient s'échanger des informations" (Alain Nys). Le responsable technique du projet doit pourtant reconnaître qu'ils ne le font pas. Et effectivement, on se demande pourquoi des enseignants qui ne sont déjà pas habitués à travailler ensemble dans une même école iraient contacter des enseignants d'une autre école. De plus, donner cours avec Internet demande un grand investissement de temps de la part des professeurs, du moins au début. Or, comme le disait un professeur de géographie, en faisant allusion au surcroît de travail imposé par l'utilisation scolaire d'Internet: "ceci est en plus du système classique" (Michel Meylemans); de nouvelles missions sont dévolues à l'enseignant, sans que celui-ci ne soit déchargé de ses tâches traditionnelles, par exemple sous forme de crédits d'heures ou d'un allègement des programmes. "On offre un peu partout aux enseignants des connexions à l'Internet qu'ils n'ont pas demandées, sans dégager de moyens supplémentaires suffisants pour aider à les mettre convenablement en œuvre. Comment faire quand, un peu partout, on n'a déjà plus tout à fait le personnel ni le matériel nécessaire pour assurer les missions traditionnelles de l'école ?"' (Michel Boumal).

De plus, un problème technique se pose : pour aller sur Internet, les écoles bruxelloises doivent passer par le serveur de "Brunette"; or, celui-ci, étant encore dans sa phase de croissance, n'est pas toujours disponible. Et lorsque c'est le cas, les professeurs se trouvent 
confrontés à des délais très longs (inhérents à Internet) pour atteindre un site ou charger un programme. La structure classique d'enseignement (par périodes de 50 minutes) se prête donc particulièrement mal à ce genre d'exercices. Michel Meylemans nous a signalé qu'après une expérience d'un an, il "ne va plus sur Internet avec ses élèves" à cause de la "lourdeur de l'aspect technique" qui l'amène souvent à ne se préoccuper que de cet aspect-là et ne lui "permet pas de faire de la géographie". Yves Bourron et al. parlent même du danger qu'il y a pour un enseignant à "pratiquer un métier qui n'est pas le sien" en étant obligé de "maîtriser plus l'informatique que la pédagogie"1.

Enfin, dernier aspect du problème, qui rejoint celui des enseignants, l'introduction réussie d'Internet à l'école impose également aux élèves de nouveaux rôles pour lesquels ils n'ont pas été formés. Alors qu'on valorise depuis leur petite enfance le travail et les résultats individuels, la passivité devant le "maître", et qu'on punit sévèrement celui qui regarde sur la feuille de son voisin, on attend soudainement des étudiants qu'ils travaillent en groupe, utilisent de façon active les nouvelles technologies, communiquent avec des condisciples d'autres pays. Nous aurons l'occasion de reparler de cet aspect du problème lors de l'analyse du projet "Parleunet".

\section{b. Une éducation au (multi)média}

Avant d'être une innovation, avant d'être une nouvelle technologie, Internet est avant tout un (multi)média, avec son langage et ses codes qu'il faut apprendre à déchiffrer.

Les concepteurs du projet insistent sur le nécessaire "esprit critique" face à Internet : "il s'agit d'éveiller le sens de la critique face

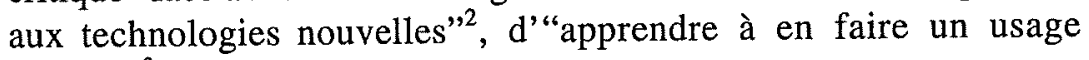
critique"3.

Mais, de la même manière qu'il y avait un flou dans la définition même du projet, il règne une incertitude quant à la définition de cet "esprit critique" : sur quoi celui-ci doit-il exactement s'exercer, et surtout, de quelle manière?

La liaison avec l'éducation aux médias n'est que rarement faite ; même dans ce cas, cette éducation semble aller de soi et s'exercer de

1 Y. BOURRON et al., op. cit., p. 180.

2 Extrait de "Le projet éducatif de la Ville de Bruxelles ", op. cit.

${ }^{3}$ Université Libre de Bruxelles - Service Télématique et Communication, op. cit., p. 1. 
façon presque automatique. Elle n'est pas considérée comme quelque chose d'essentiel à intégrer au projet, voire dont on pourrait faire l'objectif principal, au lieu de viser la maîtrise technique. Comment en effet imaginer quelque appropriation d'Internet sans éducation au (multi)média ? "Cette technologie nouvelle, avec laquelle vivront les élèves d'aujourd'hui, citoyens de demain, ne pourra véritablement leur appartenir que s'ils parviennent à l'appréhender en ce qu'elle est fondamentalement : un média" .

Seule la responsable pédagogique de la phase pilote du projet, Nathalie Messori, a insisté sur cet aspect du problème. Actuellement, il n'y a cependant plus de responsable pédagogique dans le projet... Le responsable technique du projet, Alain Nys, signale qu' "on insiste dans les formations sur l'aspect critique et les droits d'auteurs", mais affirme à propos de l'éducation aux médias que "ce n'est pas l'objectif de «Brunette»". Or, tous les spécialistes sont d'accord pour dire que l'éducation aux médias n'est pas quelque chose de "naturel" qui s'effectuerait de façon "automatique".

(...) Au niveau du regard critique et distanciateur des enfants sur les médias, l'évolution naturelle selon l'âge semble très lente. D'une manière générale, les adolescents ne prennent pas distance face à ce qu'ils regardent, d'une manière plus évidente que les enfants, l'éducation automatique aux médias ne se fait pas'.

On considère actuellement que 1'"éducation aux médias" est un véritable objet d'enseignement. Au-delà d'un "bon sens" commun à tout le monde, il faut être spécialement formé à cette matière. Celle-ci pourrait être définie comme l'"étude critique du fonctionnement et des modalités de représentation des technologies de diffusion de l'information". Ses objectifs principaux, tels qu'ils ont été définis par le "Conseil de l'éducation aux médias" en Belgique francophone sont de "former chaque élève à être un spectateur actif, un explorateur autonome et un acteur de la communication médiatique", ce qui s'obtient notamment par la "conscience d'être «recherché» par les médias comme destinataire de messages fonctionnels spécialement

${ }^{1} L$ 'école mise au net. Enjeux de l'éducation aux médias et à Internet, Bruxelles, Conseil de l'éducation aux médias, 1999, p. 20.

2 F. Thomas, Rapport d'observation et d'évaluation de "Télécole ". Une opération d'éducation des jeunes aux médias en Communauté française, Bruxelles, Fondation Roi Baudouin, 1993, p. 56. 
conçus pour produire des effets cognitifs, affectifs et comportementaux".

Malheureusement, peu de futurs enseignants bénéficient durant leurs études d'une formation adaptée, et nombre d'entre eux sont donc incapables de répercuter ce nouveau savoir auprès de leurs élèves.

Dans le cas particulier d'Internet, une éducation aux médias devrait s'opérer sur deux plans complémentaires. Tout d'abord, d'une façon traditionnelle, elle devrait mener l'élève à pouvoir "décoder", à tous les niveaux, les messages transmis par ce nouveau média. Cela va cependant plus loin : comme pour les médias traditionnels, tels que la télévision, il s'agit de faire comprendre à l'élève que ce qu'il voit "est le résultat d'une production, et non pas d'une perception"2. Les médias ne montrent pas la réalité, mais une certaine représentation de la réalité. "En devenant conscient du caractère subjectif des valeurs véhiculées, il [le jeune] acquiert la liberté d'y adhérer ou non"3.

Adhérer ou non à une certaine représentation de la réalité, à certaines valeurs : il s'agit bien là du cœur du problème, moteur d'une possible appropriation. A côté du "décodage" du message, il y a aussi, sur un autre plan, le "décodage" du medium. Nous avons déjà parlé du caractère d" "évidence" généré par le phénomène Internet, qui fait qu'on le croirait volontiers né d'une génération spontanée et se développant sans aucun contrôle. Il n'en est rien. Dominique Wolton parle par exemple du mirage d" "un individu [qui] a le sentiment de pouvoir dialoguer "naturellement" avec n'importe qui d'un bout de la planète à l'autre. En réalité, même cette communication médiatisée individuelle suppose l'existence préalable d'une infrastructure collective"4 ; cette infrastructure, prémice des fameuses "inforoutes" ou "autoroutes de l'information" dont on nous assure la venue prochaine, a été voulue, est le résultat d'un calcul politique et économique opéré par les États-Unis à la fin des années 80. "Elle est avant tout le produit de la nouvelle stratégie de croissance des États-Unis confrontés au déclin

1 L'éducation à l'audiovisuel et aux médias. Dossier de synthèse, Bruxelles, Conseil de l'éducation aux médias, 1996, p. 10

2 R. Debray, "Les aléas de la vidéosphère", in "L'éducation à l'audiovisuel : l'image de son maître ", Lettre d'information d'Eureka Audiovisuel, $\mathrm{n}^{\circ} 11$, juin 1994, p. 7.

3 Brochure de présentation des ateliers de l'asbl "Caméra Enfants Admis ", Liège, s.d., p. 3.

${ }^{4}$ D. Wolton, Penser la communication, op. cit., p. 40. 
potentiel de leur économie"1 et qui désiraient conserver la mainmise sur le commerce mondial. Le reste du monde développé, Europe en tête, a depuis lors emboîté le pas aux États-Unis, avec des buts clairement avoués de relances économique et de l'emploi : "si l'ère industrielle a eu ses grands travaux (chemins de fer, autoroutes, etc.), Internet et les autoroutes de l'information font partie du programme de grands travaux de l'ère postindustrielle"2.

Introduire Internet à l'école représente plus que la fourniture de matériel scolaire ; c'est un univers complexe qui se déploie sous les yeux des élèves, avec son langage, ses codes, ses stratégies de pouvoir, sa vision de l'avenir. On ne peut le considérer comme un simple "outil" neutre. On peut difficilement faire l'économie de discussions sur le medium avec les élèves, afin qu'ils ne soient pas que des utilisateurs passifs et plus ou moins doués, mais des utilisateurs conscients des enjeux et employant dans la mesure du possible cette nouvelle technique pour satisfaire leurs besoins propres, ce qui est sans doute la meilleure définition de l'appropriation.

\section{Deux projets utilisant Internet Les réactions des élèves}

Afin d'illustrer certains de nos propos, nous évoquerons la façon dont se sont déroulés dans deux écoles secondaires deux projets utilisant les possibilités d'Internet.

\section{a. Le projet "Parleunet"}

Le projet "Parleunet" a concerné l'Athénée Adolphe Max. Il s'agit d'un projet européen soutenu par la Commission européenne. Des élèves fréquentant des établissements secondaires de sept pays européens collaborent à la rédaction d'un rapport destiné au Parlement européen et consacré à leurs attentes en matière de travail (comment voient-ils leur avenir professionnel, désirent-ils travailler à l'étranger...). Ceci permet à la fois aux étudiants de mieux connaître le Parlement européen et aux parlementaires d'avoir une idée de ce que pensent "les jeunes".

\footnotetext{
${ }^{1}$ D. MONET, Le multimédia : un exposé pour comprendre, un essai pour réfléchir, Paris, Flammarion, 1995, p. 77.

2 A. DUFouR, Internet, Paris, PUF, 1995, p. 104.
} 
Pour ce faire, les étudiants d'une classe sont divisés en plusieurs groupes, qui vont collaborer avec leur groupe correspondant situé dans un autre pays. Dans le cas de la Belgique, des élèves de rhétorique ont coopéré avec des élèves de Suède et des Pays-Bas. Pour constituer le dossier et communiquer entre eux, les étudiants disposent des moyens techniques les plus modernes, la maîtrise de ceux-ci étant un des objectifs du projet : CD Roms, accès à une banque de données sur Internet, adresses e-mail pour chaque élève, vidéoconférence...

Nous sommes donc ici dans le cadre d'un projet très précis, aux objectifs et aux modes de déroulement bien définis. Le degré de liberté des enseignants ou des élèves est donc limité dans un certain cadre, contrairement au projet "Brunette", qui est très ouvert et non directif.

On s'aperçoit cependant que les problèmes rencontrés par le projet "Parleunet" sont d'une nature très semblable à ceux décrits pour le projet "Brunette". En effet, si des buts clairs semblent être définis, cela n'empêche pas le projet européen de partir sur des bases tout aussi friables et de ne pas être plus précis quant aux définitions de base : de quelle analyse de la réalité part-on, de quels présupposés théoriques... De plus, on ne tient pas compte du fait que les nouvelles technologies de la communication sont des médias, qui ne sont pas neutres, et des innovations, avec tout ce que cela suppose au niveau de l'adoption et de l'appropriation. L'occultation de ces aspects théoriques permet à nouveau à chacun d'investir ces nouvelles techniques de ses fantasmes propres et de plaquer sur la réalité ses espoirs ou ses illusions.

Ces différents phénomènes sont particulièrement évidents lorsqu'on analyse d'un peu plus près les paragraphes consacrés, dans le livre de présentation du projet à destination des enseignants, aux bénéfices que les élèves sont censés retirer de leur participation à "Parleunet"1. Ainsi, le deuxième paragraphe prétend que "the students will learn to communicate with people from another country and another culture". Cette affirmation part d'un présupposé aux accents déterministes : si on fournit suffisamment de techniques de communication aux étudiants, ceux-ci vont se mettre à communiquer. Le phénomène d'une communication avec des individus d'une autre culture et qui parlent une autre langue est évidemment infiniment plus

1 G. Clarebout, J. Elen, Parleunet alive. A teachers' guide for the first project intensive period, 1998, p. 79. 
complexe. Mais on se comporte ici comme si le seul obstacle à une communication réussie était le manque de technique.

Le paragraphe suivant parle du fait que les étudiants "will learn to be critical towards the «information highway»". Comme dans le cas du projet "Brunette", la façon dont les élèves devront être critiques, les domaines sur lesquels cette critique va devoir s'appliquer, ne sont pas précisés, comme si cela allait de soi, et allait apparaître naturellement, de par l'utilisation.

Enfin, le dernier paragraphe mérite d'être cité en entier, tant les ressemblances avec le texte de présentation du projet "Brunette" par le Service "Télématique et Communication" de 1'Université Libre de Bruxelles, déjà cité, sont flagrantes :

In short, the Parleunet project gives these students the opportunity to prepare themselves to become full responsible critical European citizens who will be able to function in a high technological, who will posses a cultural awareness and who will be able to work cooperatively within this multicultural, independant society.

Le même mélange d'affirmations péremptoires et de prévisions plus ou moins aventureuses se retrouve dans ce court texte : le futur y est déjà décrit, les acteurs n'ayant que très peu de capacité d'intervention. Ce qui est présenté ici correspond cependant plus au rêve de technocrates qu'à une analyse sérieuse de la réalité : les jeunes vont tout à coup se sentir des "citoyens européens", "responsables", "critiques", capables de "coopération", et travaillant dans une société "multiculturelle". Comme dans le cas du deuxième paragraphe, nous nous retrouvons dans le paradigme d'une technique agissante, certaines décisions techniques, économiques ou politiques produisant certains effets bien déterminés.

Nous avons pu assister à une séance de vidéoconférence entre les groupes d'étudiants belges et leurs pairs européens. Nos observations rejoignent les remarques ci-dessus : il y a un décalage total entre les prévisions technicistes et la réalité sur le terrain. On est encore loin des affirmations que l'on rencontre régulièrement au cours de lectures sur les nouvelles technologies de l'information: "le temps n'est pas loin où les termes de «village global», «classe mondiale», «autoroutes de l'éducation partagée»... deviendront réalités"1.

\footnotetext{
' R. COHEN, op. cit., p. 10.
} 
Pour commencer, tout comme dans le cas du projet "Brunette", la technique ne suit pas. Ainsi, sur la demi-heure dévolue à chaque groupe belge pour dialoguer avec l'autre groupe étranger correspondant, de cinq à dix minutes seulement sont disponibles pour des échanges. La qualité des images est médiocre ; le bruit de fond est très important; on ne trouve pas les bons groupes... Si le but était que les étudiants de différents pays se sentent proches les uns des autres, c'est raté. Tout cela donne plutôt l'impression de conversations avec des astronautes!

Quand enfin une connexion s'établit, cette débauche de technologie aboutit finalement à une communication sans aucun contenu. Une fois que la prouesse technique a été réalisée, on se rend compte que ces étudiants n'ont rien à se dire, et rien à s'échanger. A la question d'un groupe belge demandant à ses homologues néerlandais : "Do you read our rapport? Have you something to say?", ces derniers répondent sans complexe: "No", et la conversation s'arrête là. Et ne parlons pas d'une éventuelle collaboration... Quand le groupe néerlandais suggère à un groupe belge d'insérer dans son rapport des éléments plus personnels et moins "officiels", c'est un véritable tollé chez les étudiants belges qui n'acceptent pas du tout la critique : "We have a very good work".

Il faut convenir que les promoteurs du projet "Parleunet" signalent dans leur guide à l'usage des professeurs qu'une expérience de ce type exige l'application d'une pédagogie nouvelle, centrée sur l'apprenant. A nouveau, cependant, nous nous trouvons face à des attentes de type techniciste : l'introduction d'une technologie doit suffire pour que professeurs et élèves adoptent une autre façon de travailler. Nous avons cependant vu que les choses sont loin d'être aussi simples.

Nous laisserons le mot de la fin à la coordinatrice à Strasbourg qui, un peu dépassée par tous les problèmes techniques auxquels les groupes sont confrontés, et leur impossibilité à amorcer un dialogue, fait cette remarque non dénuée de bon sens : "if the technics does not work, maybe you can write letters to each other".

Un petit questionnaire remis à la vingtaine de participants à ce projet, à propos d'Internet et de son utilisation dans leur école, a montré que pratiquement tous les élèves possédaient au moins un ordinateur à la maison ; la moitié ont un ordinateur personnel. Dans la moitié des cas également, l'ordinateur personnel ou familial est raccordé à Internet. Pourtant, malgré une certaine utilisation à l'école 
et pour certains, à la maison, leurs connaissances théoriques sont très moyennes : beaucoup sont incapables de donner une définition d'un "lien hypertexte", certains même d'un "moteur de recherche". D'une façon théorique, tous disent savoir que les informations trouvées sur Internet ne sont pas spécialement fiables et qu'il faudra toujours les recouper avec d'autres sources. Pourtant, les professeurs remarquent de façon unanime que sur le terrain, une fois lancés sur Internet, les étudiants perdent tout esprit critique : "Internet, c'est la fascination de l'image et de l'écrit, qui acquièrent valeur de vérités totales" (Michel Van Strijtem).

A propos de leur jugement sur l'utilisation d'Internet dans leur école, à la question: "Que pensez-vous que cela vous a apporté, au niveau scolaire, d'utiliser Internet?", les étudiants répondent en priorité "cela m'a permis d'être en relation avec le monde extérieur", "j' ai appris des choses différentes de ce qu'on enseigne d'habitude à l'école" et "j'ai des rapports différents avec les autres élèves". Quand on interroge les élèves sur leurs attentes face à l'utilisation d'Internet dans leur école, la plupart espèrent en retirer "une ouverture sur le monde extérieur"; "une autre façon d'apprendre" est également souvent citée.

De telles réponses semblent indiquer un désir de renouveau de l'institution scolaire, qui devrait devenir plus ouverte, promouvoir une autre pédagogie, entraînant d'autres rapports avec les élèves. Ce désir s'investit dans Internet, mais ne perdons pas de vue que les étudiants, pas plus que les professeurs, ne se sont encore appropriés cette nouvelle technique ; tout comme les adultes, les jeunes peuvent donc prêter à Internet des caractéristiques liées à leurs propres fantasmes et espoirs d'une "autre" école.

\section{b. Le "Club Internet"}

Le "Club Internet" est une activité parascolaire qui se déroule pendant 1 heure 30, à raison d'une fois par semaine, au Lycée Émile Jacqmain. Elle réunit des élèves du cycle secondaire supérieur. C'est la première année que ce club fonctionne. Son responsable, Michel Meylemans, professeur de géographie, possédait un ordinateur à la maison, mais pas raccordé à Internet. Quand le lycée a été câblé, il s'est formé "sur le tas" et avec l'aide de collègues. En acceptant de s'occuper de cette activité parascolaire, il pensait pouvoir développer un projet avec les élèves (élaborer des "dossiers" dans des matières 
comme l'histoire, la géographie, le latin, dossiers qui auraient contenu les meilleures adresses de sites à vocation pédagogique) et évoluer techniquement en même temps que ses élèves. La moitié du temps aurait été consacré à l'élaboration de ce projet, l'autre moitié aux recherches personnelles.

Les étudiants n'ont cependant pas montré d'enthousiasme excessif pour ce mode de fonctionnement, et bien vite, le projet commun a été abandonné au profit d'une "navigation" solitaire. La demande, quand elle existe, est uniquement celle d'une maîtrise technique.

Cette expérience décevante amène quelques commentaires. Tout d'abord, il est caractéristique de noter qu'il s'agit d'une activité parascolaire. Cela montre bien la difficulté de l'école à intégrer ces nouvelles pratiques dans le cours régulier des enseignements. Le danger est évidemment que, libérés de la tutelle exercée par le professeur dans le cadre de ses leçons, les étudiants ne visent, comme on le voit ici, qu'à la maîtrise technique et ne puissent entamer une réflexion sur le sens, première amorce d'une appropriation.

Ensuite, il est également intéressant de remarquer que les étudiants, en participant à ce club, n'étaient pas demandeurs d'un projet commun et qu'ils ont préféré travailler seuls, chacun dans leur coin, alors que cette nouvelle technique est censée stimuler le travail de groupe et la coopération. Ceci confirme ce que nous savions déjà : la simple introduction d'une technique ne modifie pas pour autant les pratiques pédagogiques et l'attitude des étudiants. Les élèves ne sont pas habitués à travailler en groupe ; ce n'est pas en leur laissant accès à Internet qu'ils vont soudainement le faire.

Le petit questionnaire remis à la quinzaine de participants au "Club Internet" fait apparaître qu'un seul élève n'a pas d'ordinateur chez lui. Dans la plupart des cas cependant, l'ordinateur, relié à Internet, est celui des parents. Michel Meylemans nous a signalé qu'il avait l'impression que bon nombre d'étudiants participaient à l'activité parascolaire afin d'éviter de trop utiliser l'ordinateur familial.

Les connaissances théoriques à propos d'Internet sont relativement faibles, même si la maîtrise technique est importante.

Interrogés sur leurs attentes face à l'utilisation d'Internet dans leur école, les réponses les plus souvent citées sont "une autre façon d'apprendre" et "un divertissement". Ici aussi, un certain désir de renouveau semble se manifester, avec toutes les réserves déjà émises. Vient ensuite comme attente "un apprentissage de l'utilisation de 
l'informatique (pour un futur usage professionnel)", qui est à rapprocher du désir de maîtrise technique de l'outil manifesté par ces étudiants, maîtrise censée les aider sur le marché du travail. On ne peut que constater que le cadre théorique et l'expérience de l'école permettraient d'aller beaucoup plus loin...

\section{Conclusion}

Internet à l'école est plus qu'une technique nouvelle, qu'un simple outil nouveau, c'est une innovation. Et avant d'introduire une innovation dans l'univers de l'enseignement, il est essentiel de résoudre différents problèmes de définitions. Qu'est-ce exactement qu'Internet, d'où vient cette nouvelle technologie, quelle est sa logique de développement, quelle vision de la société véhicule-t-elle, quels sont ses usages actuels, possibles, ou annoncés, de quelle manière cette innovation peut-elle répondre à nos besoins, quels sont les usages que nous, utilisateurs, envisageons, dans quel projet cela va-t-il s'insérer, que recherche-t-on exactement via l'introduction de l'innovation?

Répondre à toutes ces questions, c'est mettre en place ce qui manque le plus au projet "Brunette" pour l'instant, à savoir un projet pédagogique global et, Internet étant aussi, et surtout, un (multi)média, un programme cohérent d'éducation aux médias.

A ce moment seulement, le lent processus de l'appropriation, sans lequel l'introduction d'Internet au sein de l'école n'a ni beaucoup de sens, ni beaucoup d'utilité, peut s'amorcer.

Et pourtant, s'"il y a du pain sur la planche pour les différents acteurs du système éducatif (...), l'obligation est ardente et l'aventure passionnante". Comme le disait un professeur, "qui d'ailleurs pourrait longtemps nier son incroyable richesse intrinsèque ? Éduquer par l'Internet, ce n'est peut-être pas facile à mettre en œuvre, mais c'est se doter d'un tel potentiel éducatif !" (Michel Boumal).

Le projet "Brunette" est très jeune, pas encore achevé à 1'heure où nous écrivons ces lignes. Le présent article doit donc être considéré comme un premier bilan, provisoire.

Le projet a tous les défauts d'un plan pluriannuel dûment planifié, dont les principaux sont certainement l'absence d'un projet péda-

${ }^{1}$ B. DizAmbourG, "Éditorial ", Internet dans le monde éducatif, op. cit., p. 2. 
gogique global, le manque de reconnaissance d'Internet en tant que (multi)média et des objectifs très flous. Il a néanmoins aussi les "qualités de ses défauts". Pour une fois, on n'assiste pas à des solutions de "bricolage": il s'agit d'un projet sérieux, stable dans la durée, dans lequel toutes les écoles sont équipées, tous les frais sont pris en charge, les formations des personnels assurées...

Ces différentes qualités permettent parfaitement de rectifier le tir. Un audit exhaustif de la situation dans les différents établissements devrait être effectué, grâce auquel tous les acteurs (concepteurs, responsables politiques, préfets, professeurs, élèves...) pourraient ensemble décider du sens qu'ils entendent donner à l'introduction de cette nouvelle technologie et quels sont les buts précis qu'ils lui assignent.

Ces deux éléments forment certainement ce qui a le plus fait défaut à tous les plans ou projets d'équipement des écoles en technologies considérées comme novatrices à l'époque, comme la télévision, la vidéo, et surtout l'informatique.

Pour que le projet "Brunette" puisse réussir, il faut lever les incohérences et les ambiguïtés qui sont encore présentes et assurer une complète cohérence interne, que ce soit au niveau des définitions préalables, des buts recherchés, de la (nouvelle) pédagogie à mettre en œuvre...

Pendant ce temps, la perception que l'on pouvait avoir de l'outil Internet est en train de se modifier. D'un moyen d'accès à des informations en provenance du monde entier, il est de plus en plus perçu comme une technologie assumant des fonctions ludiques et communicationnelles.

La question : "Quel Internet veut-on pour quelle école ?" devient donc plus que jamais cruciale si l'on désire que les professeurs et les élèves s'approprient réellement cette nouvelle technologie. 